\title{
EDUCACIÓN EN ABIERTO: INTEGRACIÓN DE UN MOOC CON UNA ASIGNATURA ACADÉMICA
}

Ángel
FIDALGO
BLANCO
Universidad Politécnica de
Madrid
Escuela de Minas y
Energía
Departamento de
Ingeniería Geológica y
Minera
Ríos Rosas, 21
28003-Madrid
angel.fidalgo@upm.es

Ángel

María Luisa

SEIN-

ECHALUCE

LACLETA

Universidad de Zaragoza

Escuela de Ingeniería y

Arquitectura

Departamento de Matemática

Aplicada

María de Luna, 3

50018-Zaragoza

mlsein@unizar.es

\author{
Oriol BORRÁS \\ GENÉ \\ Universidad Politécnica de \\ Madrid Rectorado \\ Gabinete de \\ Tele-Educación \\ Ramiro de Maeztu, 7 \\ 28040-Madrid \\ oriol.borras@upm.es
}

Francisco José

GARCÍA

PEÑALVO

Universidad de

Salamanca

Instituto Universitario

de Ciencias de la

Educación

Departamento de

Informática y

Automática

Grupo GRIAL

fgarcia@usal.es

\section{Resumen:}

La educación en abierto comienza a consolidarse con la aparición de los OER (Open Educational Resources), recursos de aprendizaje en abierto y accesibles a través de internet. El modelo OCW (OpenCourseWare) se implanta fuertemente en el contexto universitario y, actualmente, los MOOC (Massive Open Online Curse) constituyen el máximo exponente de la educación en abierto. Se muestra cómo la interacción entre los OER pertenecientes a distintas iniciativas: OCW, MOOC y Redes Sociales, para una misma asignatura académica, mejora tanto la propia asignatura como los OER involucrados. El modelo presentado incorpora a la asignatura los recursos de aprendizaje de un MOOC: material del profesorado, red social profesional dentro del MOOC y recursos en abierto generados por los participantes del MOOC. Para validar el modelo se analiza el impacto en el alumnado de la incorporación de los recursos del MOOC en la asignatura académica, con unos resultados muy positivos.

Palabras clave: Recursos educativos abiertos, Aprendizaje en línea, Aprendizaje Social, Curso en línea abierto masivo. 


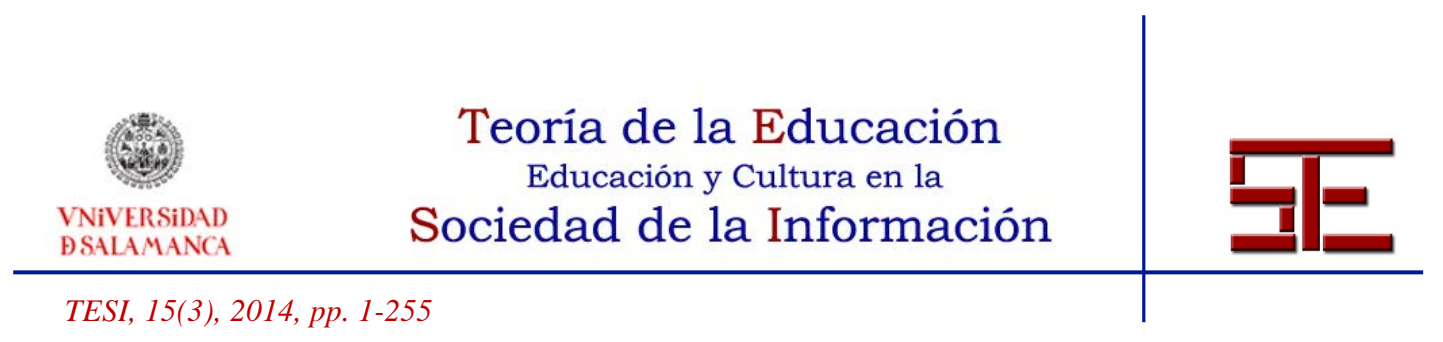

\section{OPEN EDUCATION: INTEGRATION OF A MOOC WITH AN ACADEMIC} SUBJECT

\section{Summary:}

Open education begins to consolidate with the OER (Open Educational Resources) appearance, which are open learning resources and accessible on internet. The OCW (OpenCourseware) model is strongly implanted in the university context and the MOOC (Massive Open Online Courses) are currently the greatest exponent of open education. This paper is deveoted to show how the interaction between different OER initiatives: OCW, MOOC and Social Networking, for the same academic subject, improves both the subject itself and the involved OER. The model, presented here, integrates the MOOC learning resources: faculty material, professional social networking inside MOOC and open resources generated by MOOC participants. In order to validate the model, the impact on students, after the inclusion of MOOC resources in academic subject, is analyzed with very positive results.

Key words: Open Educational Resources, Online learning, Social learning, Massive Online Open Course. 


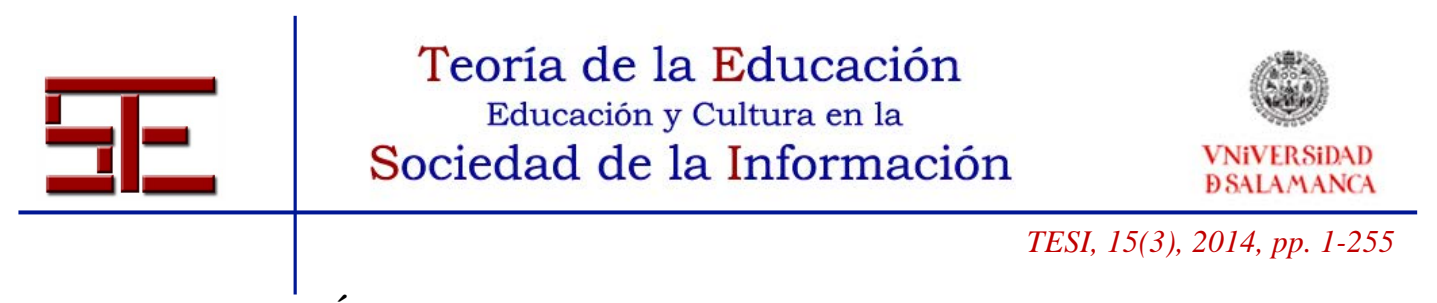

\section{INTRODUCCIÓN}

El movimiento en abierto se inicia con el software libre y de código abierto, donde las ideas principales se basan en la compartición de recursos, el trabajo cooperativo y en la libre utilización y transformación del código fuente del software. Este movimiento en abierto se extiende a diversos sectores, entre ellos el educativo (García-Peñalvo et al., 2010a, 2010b).

La comunidad universitaria comienza a distinguir entre código abierto (principal baluarte del movimiento en abierto) y los materiales formativos en abierto (Moore, 2002). Los recursos en abierto para la educación se denominan Open Educational Resources (OER) (UNESCO, 2012) y, a partir de los mismos, surge el movimiento OER, que hereda los principios y las libertades del software libre y que, de forma progresiva, va definiendo una serie de necesidades, como:

- La integración entre herramientas (sistemas eLearning, software social, sistemas de gestión de contenidos y herramientas de desarrollo), contenidos y recursos (licencias, buenas prácticas, etc.) desde el punto de vista técnico (Margulies, 2005).

- Tener en cuenta las características técnicas, las características sociales y el área de conocimiento del propio recurso (CNI, 2008).

- La reutilización de los recursos en una gran variedad de situaciones de aprendizaje (Wiley, 2006).

El impacto del movimiento OER se vislumbra de gran potencial para cambiar el método de aprendizaje (McAndrew, 2010), originando un cambio radical en las necesidades de los agentes relacionados con la formación (Holmes, 2006).

Actualmente los OER se asocian a una nueva cultura basada en la cooperación (Ossiannilsson y Creelman, 2011), a la trasformación de los roles del profesorado como mentor, facilitador y tutor del conocimiento y sus fuentes (UNESCO, 2011a, 2011b). Asimismo, busca conseguir varios retos como la implicación de los estudiantes en la elaboración y reutilización de contenidos en abierto (UNESCO, 2010), centrar la innovación en los procesos y en las personas (Lane, 2010), crear comunidades activas de aprendizaje (Liddo et al., 2012), el intercambio de recursos educativos para su reutilización y la sostenibilidad de los mismos (McAndrew, 2012).

Los OER son considerados por organismos como la OECD (2007) o la UNESCO (2011b) como uno de los principales desafíos a los que se enfrenta la educación superior con implicaciones e impacto en el modelo de aprendizaje, estratégico y económico de la universidad.

En este contexto han surgido iniciativas OER corporativas; OpenCourseWare (OCW)

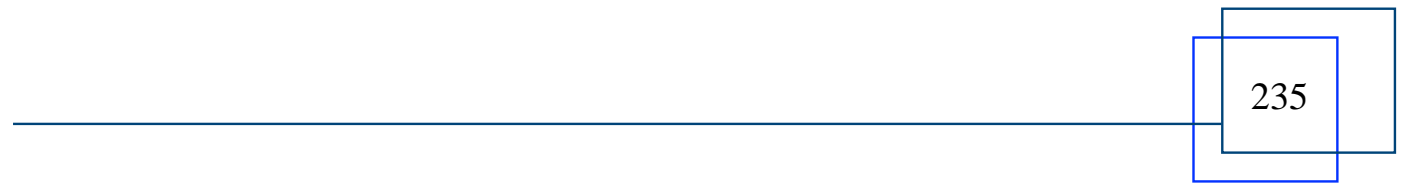




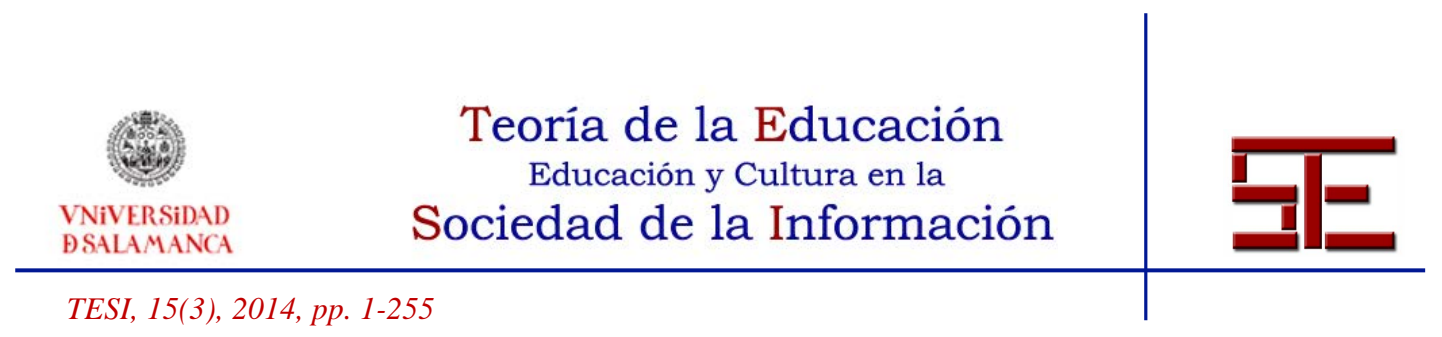

(OCWC, 2014) se puede considerar como la primera iniciativa OER que arranca cuando el Instituto Tecnológico de Massachusetts (MIT) anuncia en 2001 que dará acceso gratuito a sus materiales educativos en forma de asignaturas, correspondientes a sus estudios universitarios. Desde que en 2002 lanzó las primeras 50 asignaturas ya cuenta con 2150 publicadas (MIT, 2014).

Siguiendo la senda de OCW hay otras iniciativas que han dado un paso más en la utilización y consolidación del conocimiento en abierto como son: la Khan Academy (Khan, 2006) y los MOOC (Massive Open Online Courses) (Downes, 2008; Markoff, 2011) todas ellas orientadas a la educación en abierto.

La web social no se ha quedado atrás, y basada en ella, han surgido cientos de iniciativas OER utilizando redes sociales, blogs y wikis. Asimismo se ha consolidado una teoría de aprendizaje en lo social, y Siemens (2006) define la teoría del conectivismo, uniendo más tarde iniciativas corporativas como los MOOC con la web social en los denominados cMOOC.

Por otra parte, el movimiento OER ha generado iniciativas corporativas y sociales a partir de cursos tradicionales impartidos en las universidades. No obstante, en la actualidad hay una serie de barreras:

- Los OER generados a partir de cursos tradicionales avanzan hacía nuevos modelos de aprendizaje. En cambio, esos mismos cursos, dentro de la universidad, no han tenido impacto en la trasformación del aprendizaje.

- La usabilidad, reutilización y sostenibilidad de los contenidos tienen enfoques dispares. Mientras en los OER de la web social se hace de una forma dinámica y continua, en iniciativas corporativas como OCW tienen un ciclo de actualización muy lento.

- El desarrollo de comunidades de aprendizaje, formadas por alumnado, profesorado, profesionales y organizaciones no están funcionando.

- No existe cultura de colaboración entre los alumnos y usuarios de los OER, principalmente en la formación académica.

Este trabajo se enmarca en una línea de investigación que busca integrar la formación académica con la formación en abierto y concretamente, con los últimos modelos de formación en abierto, como los OCW y MOOC. Se muestra cómo la interacción entre los OER pertenecientes a distintas iniciativas: OCW, MOOC y web social (redes sociales y wiki), todos ellos vinculados a una misma asignatura, mejora tanto la propia asignatura como los OER de las distintas iniciativas.

En las siguientes secciones se describe el uso de los OER en una asignatura académica que genera nuevos OER. A continuación se describe el OCW que se generó a partir de

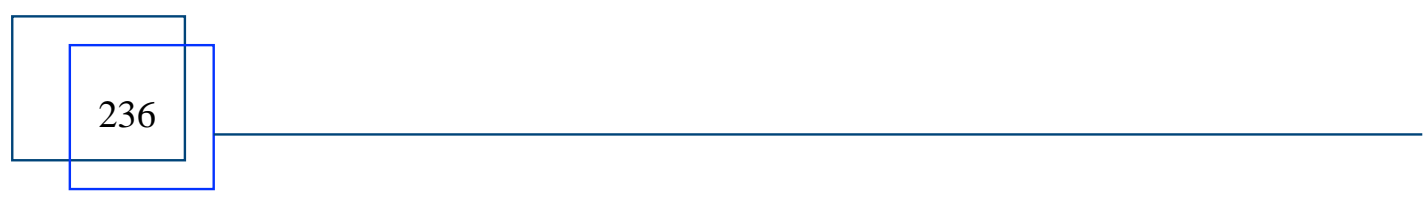




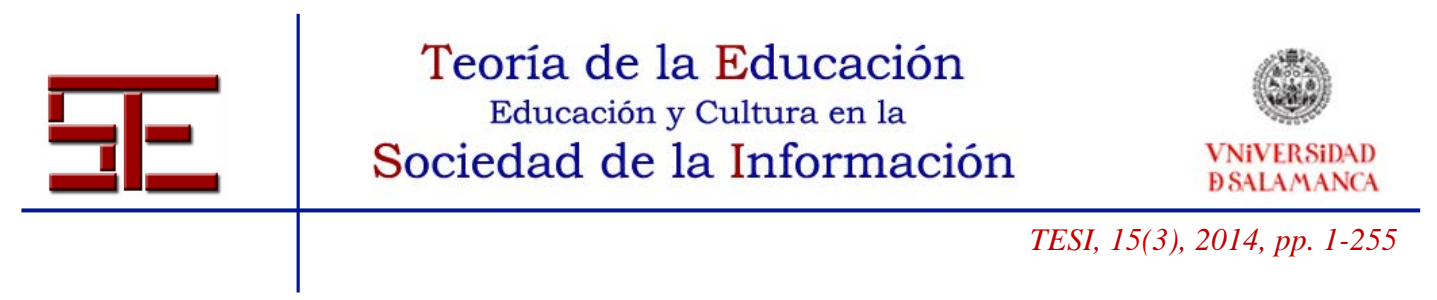

la asignatura académica, seguido del MOOC realizado a partir de los OER de dicha asignatura. El MOOC genera, a su vez, OER en la web social y estos se utilizan de nuevo en la asignatura académica. Se muestra el desarrollo del modelo propuesto basado en un espiral de conocimiento abierto que integra las distintas iniciativas OER con la asignatura académica. Se incluyen los distintos resultados obtenidos de la experiencia $\mathrm{y}$, entre ellos, una encuesta de satisfacción por parte del alumnado. Finalizando con las conclusiones y líneas de trabajo futuras.

\section{DEL OER AL MOOC}

Los OER se pueden clasificar por la forma de interactuar los usuarios de los mismos; es decir la integración del modelo producer-consumer (ej. OCW) y el de co-production (ej. wiki, web social) (Piedra, 2010). El modelo se basa en la integración de esos dos modelos a partir de la actividad de los usuarios organizándose en comunidades de prácticas que integran los modelos anteriores con los modelos de formación académica y social (MOOC), citando a Pisutova (2012) "Openness and sharing has always been part of education process".

En esta sección se muestra la adecuación de una asignatura dentro de la oferta oficial de una universidad, que ha evolucionado al incluirla en diferentes iniciativas OER (OCW, MOOC y web social), como se refleja en la Figura 1.

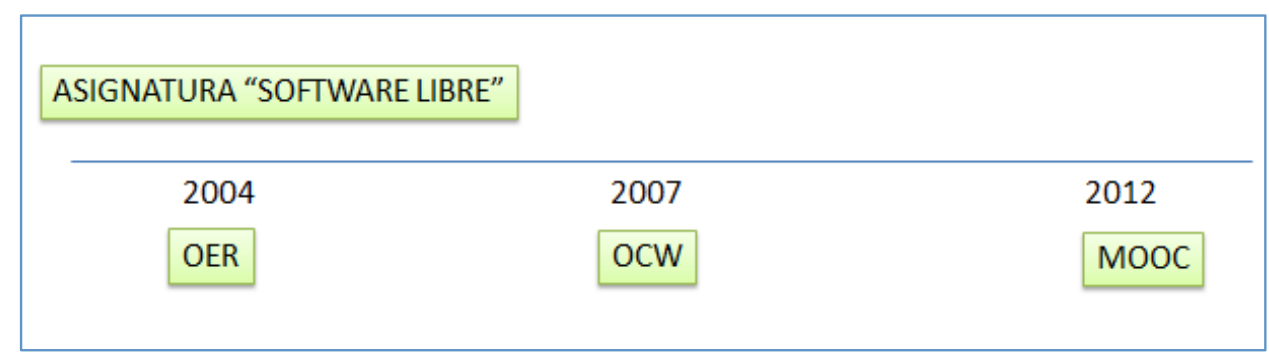

Figura 1. Evolución de una asignatura en distintos modelos del movimiento educativo en abierto.

\subsection{La asignatura académica basada en los principios del movimiento OER}

En el año 2004 el Laboratorio de Innovación en Tecnologías de la Información (LITI) implanta la asignatura académica "Software libre" (totalmente online) en la Universidad Politécnica de Madrid. Esta asignatura es de libre elección (hasta 2010-2011) para todas las universidades públicas de Madrid (dentro del proyecto ADA-Madrid (ADA-Madrid, 2014) en el curso 2011-2012 fue la única asignatura de la UPM que se puso bajo la normativa de Bolonia y en el curso 2012-2013 es asignatura de libre elección de la

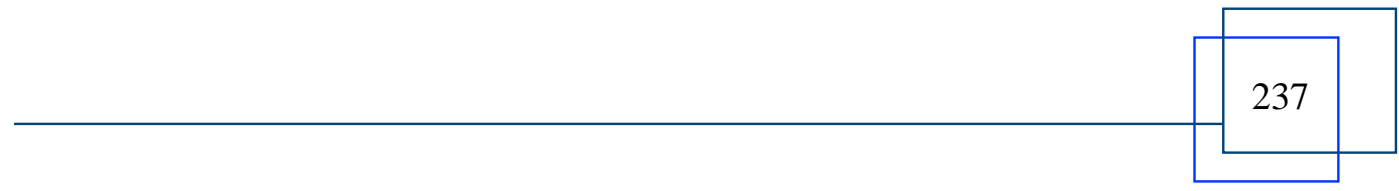




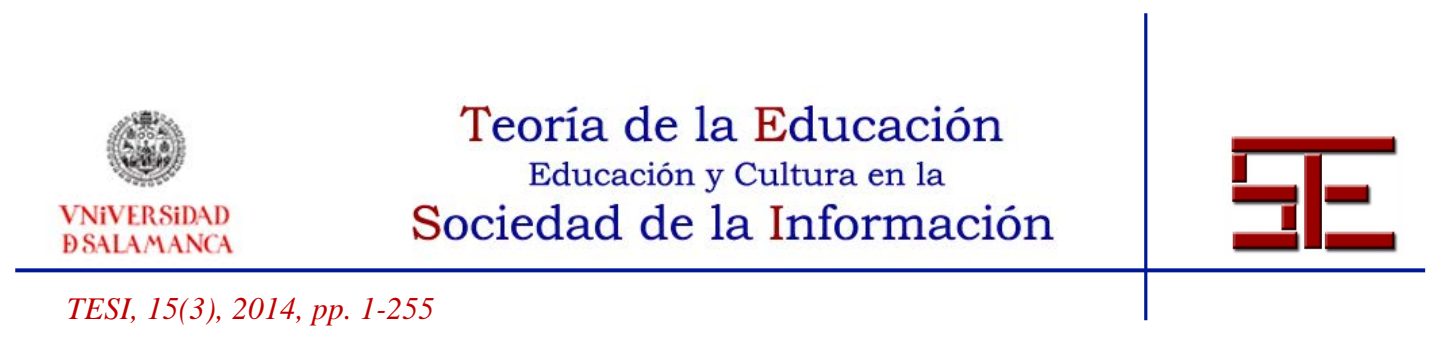

UPM y la Universidad Autónoma de Barcelona (UAB).

Para la implantación de esta asignatura, se crea un modelo de aprendizaje en el que se intenta incluir la mayor parte de las características que posee el entonces incipiente movimiento OER y en especial las tres siguientes:

3. Conocimiento en abierto. La utilización de conocimiento en abierto (el generado por el profesorado y el externo) en los materiales de aprendizaje de las asignaturas.

3. Disponibilidad y accesibilidad al conocimiento. Para que los estudiantes pudiesen acceder al conocimiento de las lecciones magistrales, y no quedase limitada al aula, se utilizó ya una técnica, que dos años después se denominó flip teaching, sacando fuera del aula las clases de corte teórico a través de vídeos y centrando la actividades de aprendizaje a través de la interacción entre estudiantes y profesores. Todo el conocimiento (archivos y vídeos) de esta asignatura se incluyó en la plataforma de eLearning Moodle (Moodle, 2014) y las actividades cooperativas en BSCW (para actividades asíncronas) (BSCW, 2014 ) y NeetMeeting (para actividades síncronas) (NetMeeting, 2014).

3. Conocimiento en abierto como bien público. Hacer partícipe a la sociedad, y no solo a los estudiantes, del conocimiento generado en la asignatura (principalmente el generado por los estudiantes). Para ello se realizó una versión electrónica del concepto de "Aprendizaje Servicio" (Aps, 1993) que se basa en que los recursos, que eran libres y generados en la asignatura, se consideraban como un servicio público. Por tanto, esos recursos debían salir fuera de las fronteras espaciales (del campus) y temporales (del período de docencia). Es decir, eran válidos y mejorables en tiempo y lugar.

Pero a pesar de la aplicación de los mismos principios que los expresados años después por Wiley, (2006), Khan (2006) y OECD (2007), la asignatura presentaba varias barreras (puntos débiles) que impedían aplicar los principios del movimiento del conocimiento en abierto y las principales fueron:

- No accesibilidad permanente del material de aprendizaje. Los contenidos generados a través de wikis y blogs eran accesibles una vez finalizada la asignatura y por personas ajenas al curso. Sin embargo, el material de aprendizaje colocado dentro de la plataforma Moodle de la universidad no permitía el acceso, ni siquiera, a los propios estudiantes una vez finalizado el curso.

- Falta de implicación social en el desarrollo de la asignatura. Es decir, la imposibilidad de permitir la participación de personas que no fuesen de la asignatura en la mejora de los recursos de la misma.

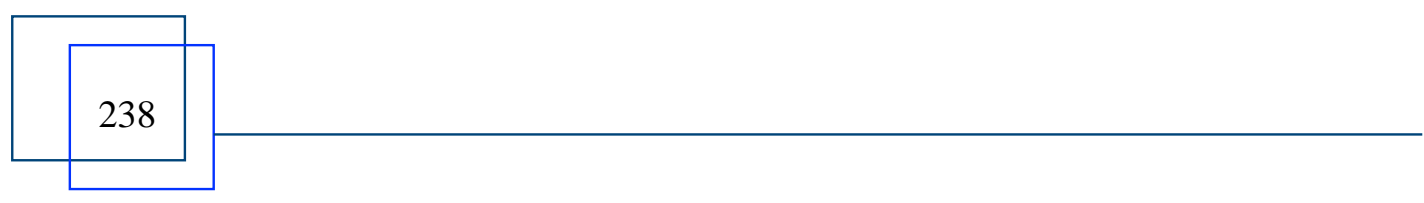




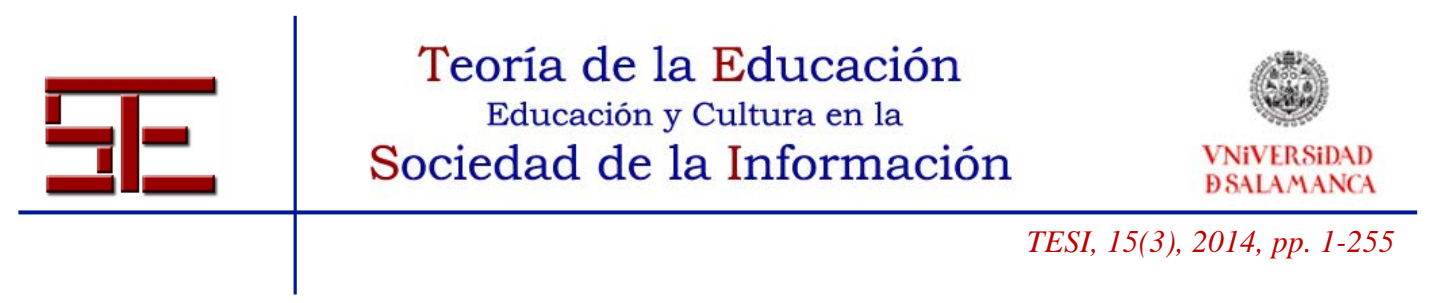

En la siguiente fase, la incorporación de la asignatura al OCW de la UPM consigue eliminar la primera barrera y atenuar la segunda.

\subsection{OCW como catalizador del movimiento OER en la asignatura académica}

En el año 2007 comienza la iniciativa OCW en la Universidad Politécnica de Madrid. En ese mismo año se incluyó en OCW la asignatura "Software libre" (SL, 2007) con el objetivo claro de socializar todo el contenido de aprendizaje de la misma. La inclusión de la asignatura en OCW tenía por objeto sumarse a la iniciativa, pero también solventar las barreras con las que se topaba la asignatura.

No solo se trasladaron a OCW los contenidos de la asignatura académica sino el modelo formativo y las innovaciones de la asignatura como el flip teaching (con los vídeos y las actividades participativas), así como la proyección social de los contenidos de la asignatura a través de wikis que permanentemente están abiertos y modificados por los alumnos de la asignatura. Estas características dotan a la asignatura OCW "Software libre" de un dinamismo basado en la metodología, las innovaciones y el enfoque de la propia asignatura.

La demostración palpable de estas afirmaciones y características expuestas están avaladas por la concesión, en 2008, del primer premio en la primera convocatoria del premio Ministerio de Educación-Universia a la mejor asignatura en OCW, al profesor de la UPM Ángel Fidalgo, con la asignatura "Software libre".

Se presentaron 55 asignaturas entre nueve universidades españolas. En primera ronda se escogieron por votación las asignaturas preferidas por más de 1.000 estudiantes registrados en Universia. La resolución del premio corrió a cargo de un jurado presidido por el rector de la Universidad de Sevilla, un representante del Ministerio de Educación y tres vocales miembros del consorcio mundial de OCW (OCW, 2008).

La mayor parte del profesorado universitario que incluye una asignatura en OCW lo hace por apoyar el movimiento OER y al propio consorcio OCW. En el caso que aquí se presenta, el traspaso se realizó, además, para analizar la forma en que OCW podía ayudar a mejorar el aprendizaje de la asignatura y, al mismo tiempo, avanzar en el modelo OER de la misma. En este sentido OCW contribuyó a mejorar el modelo de innovación y de aprendizaje de la asignatura.

\subsubsection{Mejora del modelo de innovación de la asignatura}

El enfoque de servicio de la asignatura se vio impulsado por OCW. Los wikis desarrollados en la asignatura y accesibles a través de OCW incremento la visibilidad de los mismos considerablemente y comenzaron a llegar comentarios y peticiones de

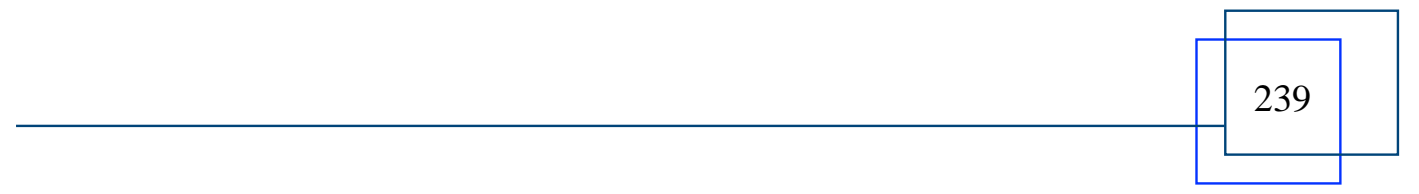




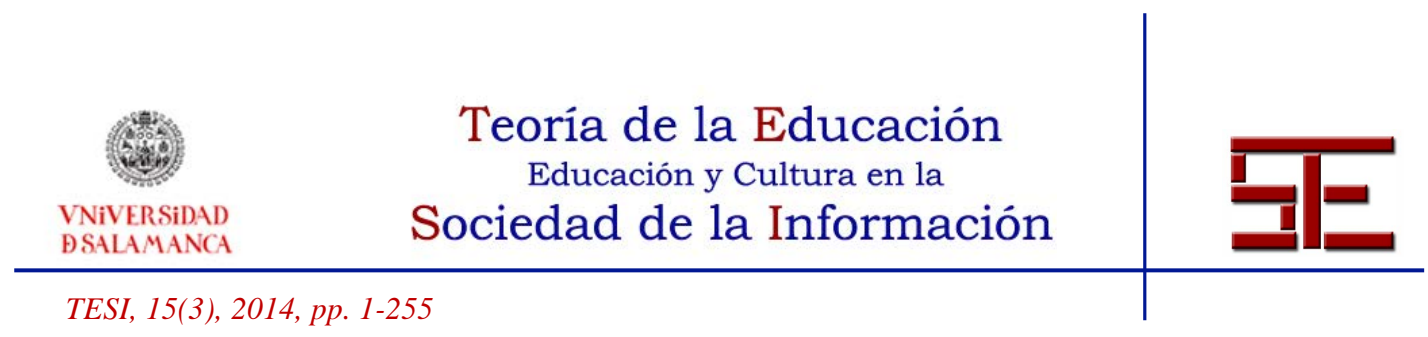

participación en el mismo de personas ajenas a la asignatura.

\subsubsection{Mejorar el modelo de aprendizaje de la asignatura}

Se consiguió eliminar las barreras del campus, permitiendo que los contenidos de aprendizaje fuesen accesibles de forma permanente, lo que permitió dar un servicio de pre-aprendizaje, durante el aprendizaje y en el post-aprendizaje.

- Pre-aprendizaje. Permite a los estudiantes acceder a los contenidos, actividades, programa de la asignatura académica (optativa) antes de matricularse.

- Aprendizaje. Se permite acceder y trabajar directamente con la asignatura OCW, simultáneamente a la asignatura oficial y su estructura y forma de organizar la información hace que sea más cómodo consultar los contenidos de aprendizaje a través de OCW.

- Post-aprendizaje. Supuso un elemento motivacional importante para los alumnos, ya que sabían que una vez finalizada la asignatura podían acceder a los contenidos.

El éxito de la repercusión de OCW en la propia asignatura sirvió de catalizador para abrir nuevos wikis con contenidos tanto de OCW como de la asignatura académica. De esta forma se impulsó tanto el servicio público de la asignatura como el aprendizaje informal. A modo de ejemplo se resalta el wiki "Creando wikis" (Wiki, 2014). Con más de 600.000 visitas, cerca de 1.000 personas han solicitado participar en el wiki y más de 700 personas han contribuido a mejorar el wiki enviando sus propuestas de mejora y cuestionarios sobre el uso de esos recursos.

La inclusión de la asignatura académica en OCW contribuyó a mejorar el modelo de innovación de la asignatura, a dar más alcance al modelo de aprendizaje e impulsar el modelo de servicio público y formación informal pero aún quedaba pendiente una de las características principales: la creación de conocimiento en abierto a partir de comunidades de aprendizaje utilizando redes sociales.

A pesar del impacto de OCW en la sociedad, sus cursos no permiten organizarla en comunidades de aprendizaje ni utilizar el conocimiento de los usuarios de OCW para mejorar la propia asignatura. Esto se debe principalmente a dos grandes barreras que presenta actualmente:

- Lenta actualización de contenidos. La actualización de contenidos es lenta, llegando a requerir realizar solicitudes, contratos y esperar un largo tiempo a que se formalice. En una asignatura con una metodología basada en el movimiento

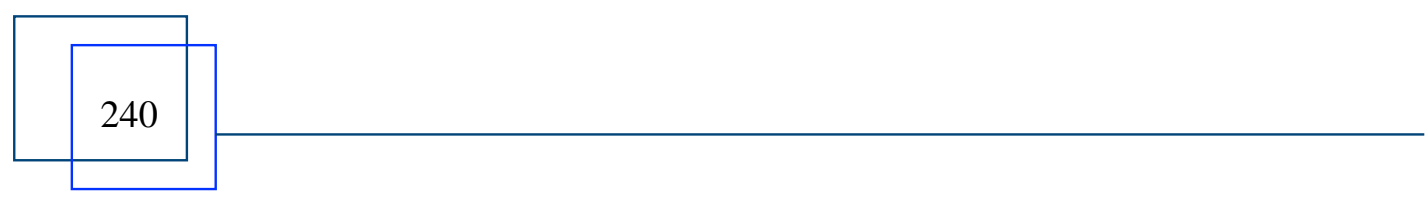




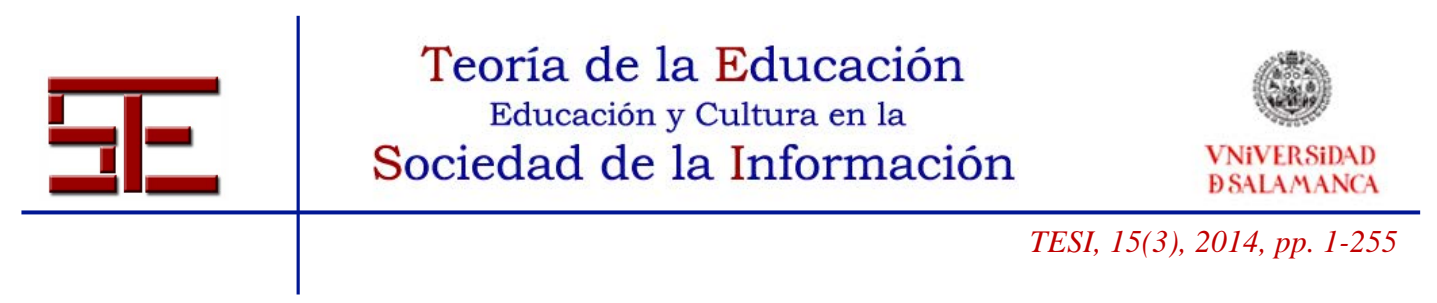

del conocimiento en abierto y en los estudiantes como generadores de conocimiento, los contenidos se actualizan todos los años; por tanto se produce un desfase entre los contenidos de la asignatura académica y los contenidos de OCW. Este mismo problema se acentuaba en el wiki al que enlaza el OCW, los contenidos que se enlazan desde el propio OCW están mucho más actualizados respecto a los estáticos de la asignatura OCW.

- Imposibilidad de interactuar con los usuarios de OCW. Solo se obtienen datos de acceso a los contenidos del curso OCW pero no se puede medir el impacto del uso de esos contenidos en el aprendizaje.

Por todo lo anterior, el equipo de trabajo consideró la posibilidad de incorporar comunidades de aprendizaje al modelo en su fase de OCW.

\subsection{MOOC: La última evolución del OER}

En función de la tecnología, la estrategia didáctica y el nivel de cooperación podemos encontrar dos tipos de MOOC: los xMOOC y los cMOOC. Las principales características y diferencias se recogen en la Tabla 1 (Fidalgo et al., 2013b).

Tabla 1. Características y diferencias entre xMOOC y cMOOC.

\begin{tabular}{|l|l|l|}
\hline & xMOOC & cMOOC \\
\hline $\begin{array}{l}\text { Tecnología: } \\
\text { Similitud } \\
\text { con: }\end{array}$ & $\begin{array}{l}\text { Cursos online (Learning } \\
\text { Management Systems, } \\
\text { LMS) }\end{array}$ & $\begin{array}{l}\text { Redes Sociales y entornos } \\
\text { personales de aprendizaje }\end{array}$ \\
\hline $\begin{array}{l}\text { Estrategia } \\
\text { didáctica }\end{array}$ & $\begin{array}{l}\text { Conductista } \\
\text { Formación formal } \\
\text { Centrada en contenidos } \\
\text { Evaluación (test, entrega } \\
\text { de trabajos, evaluación por } \\
\text { pares) }\end{array}$ & $\begin{array}{l}\text { Conectivista } \\
\text { Formación informal } \\
\text { Centrada en tareas } \\
\text { Evaluación (conocimiento } \\
\text { personales de aprendizaje) }\end{array}$ \\
\hline Cooperación & En la evaluación y en foros \\
\hline
\end{tabular}

Los cursos más abundantes son los de tipo $\mathrm{X}$, debido a su similitud con los cursos

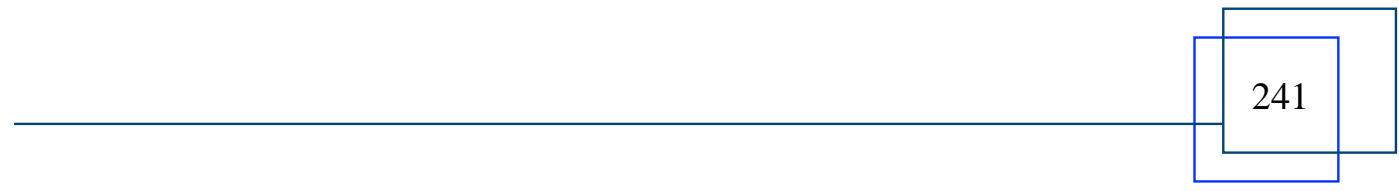




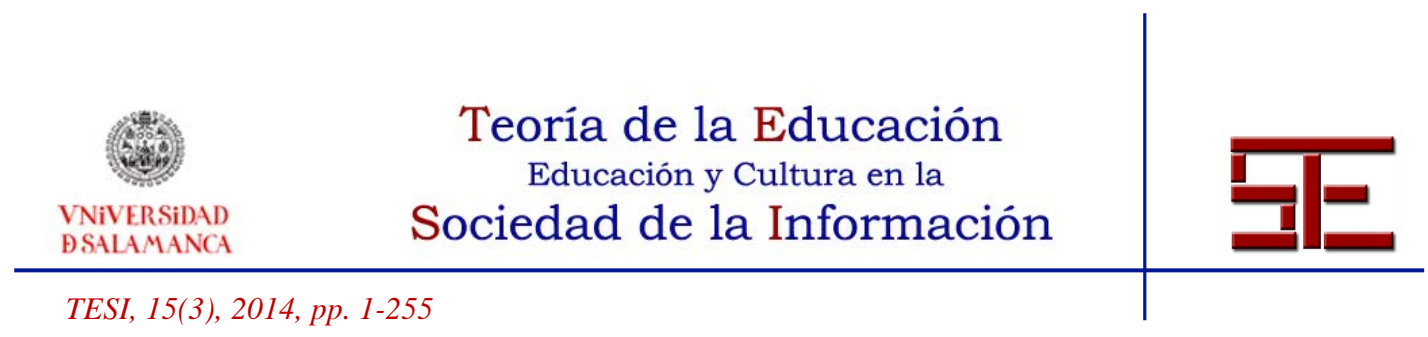

online tradicionales y por el número de plataformas que se basan en él (García-Peñalvo, 2005; García-Peñalvo, 2008). Los de tipo C son mucho más escasos, debido a la dificultad de organizar el contenido y los recursos de aprendizaje que generan los participantes en sus propios entornos personales.

El modelo MOOC tiene un enfoque social, potencia las comunidades de aprendizaje, rompe con el modelo formativo tradicional y se integra de lleno en el movimiento OER. Es por ello que en diciembre de 2012 se crea el MOOC "Software Libre y Conocimiento en abierto" (MOOC, 2012) en la plataforma MiríadaX y que fue impartido del 11 de marzo al 23 de abril de 2013.

Se incluye la misma metodología que en la asignatura académica y el OCW, por tanto todos los OER tienen el mismo planteamiento metodológico. En esta ocasión, para enriquecer las grabaciones, se ha optado por mezclar mediante una técnica de chroma keying dos elementos multimedia, por un lado el docente y por el otro un fondo en el que mediante dicha técnica con una mezcladora de vídeo se pueda ver la captura de pantalla de aquello que está explicando el docente, o cualquier tipo de presentación a modo de refuerzo (Borras, 2012).

\section{MODELO TEÓRICO: ESPIRAL EN ABIERTO. INTEGRACIÓN DE LOS OER, OCW Y MOOC}

Los modelos OER, OCW y MOOC, se pueden considerar como la evolución del movimiento en abierto, por tanto actualmente se utiliza el más evolucionado (el MOOC); sin embargo, nuestro modelo se basa en realizar una espiral continua entre los diversos modelos que actualmente existen.

Como muestra la Figura 2, la idea principal es que se establezca un flujo continuo entre la asignatura académica, el OCW y el MOOC. El nexo de unión son las redes de aprendizaje (generadas por el MOOC) y el conjunto de OER que se van actualizando y ampliando a través de la asignatura, el OCW y el propio MOOC.

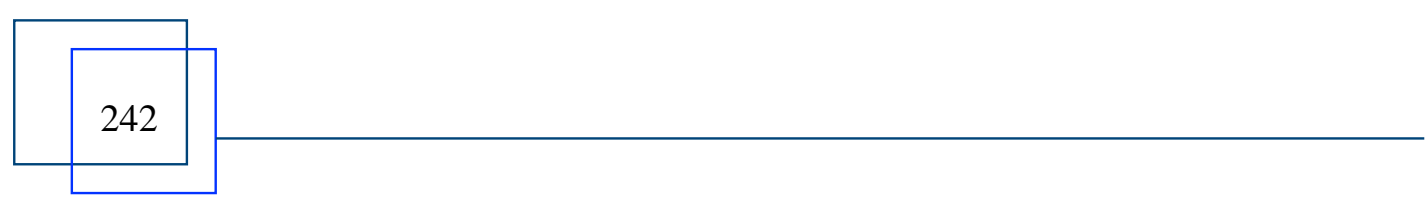




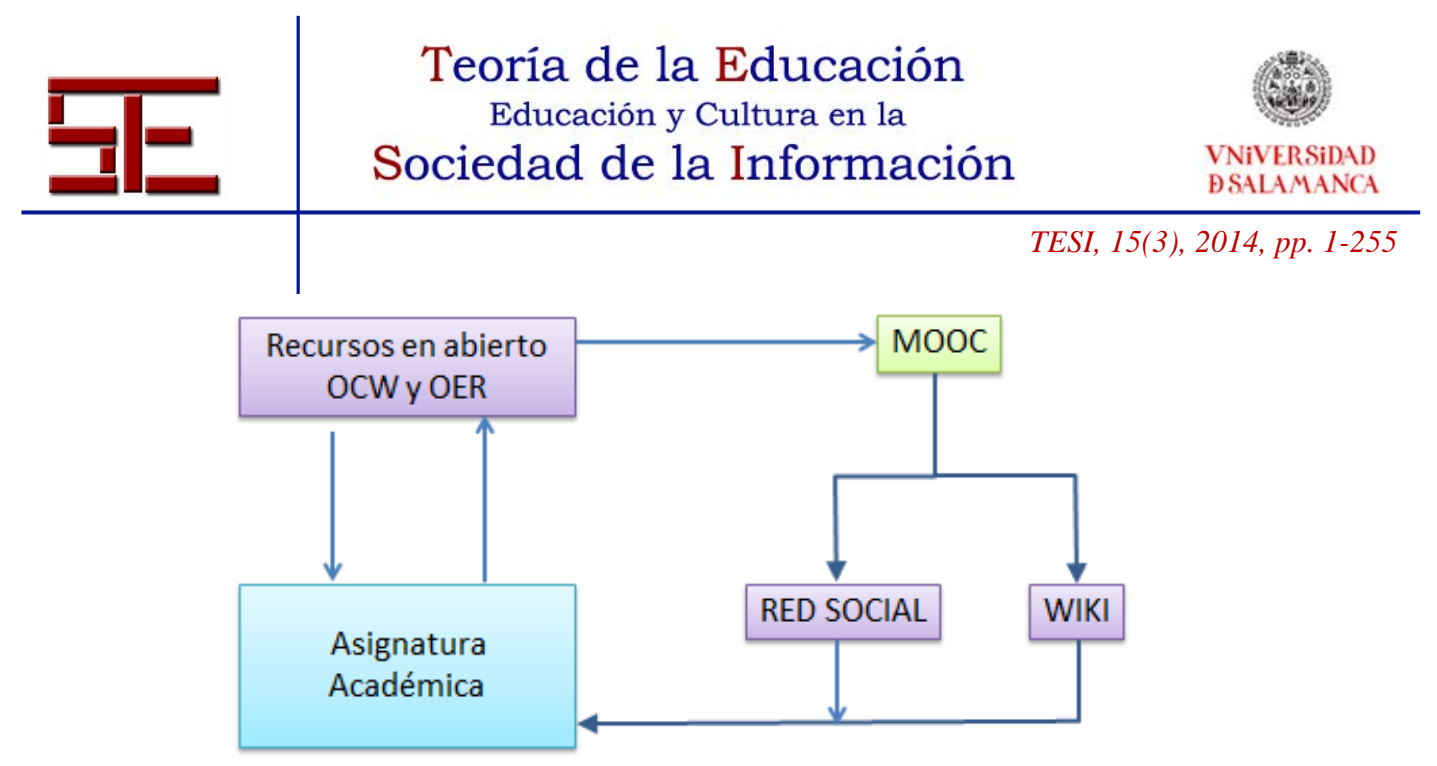

Figura 2. Modelo de integración entre distintas iniciativas OER con una asignatura académica.

Los OER generados en la asignatura académica se utilizan en el OCW y, a su vez, en el MOOC. El MOOC genera nuevos OER que se integran en un wiki y en una red social. La red social y el wiki se integran en la asignatura, lo que permite realizar nuevos planteamientos de aprendizaje.

Actualmente la asignatura dispone de los siguientes recursos del MOOC:

- OER generados por el profesorado (vídeos profesor).

- OER generados por colaboradores pertenecientes al mundo profesional y empresarial (vídeos colaboradores).

- OER sociales. Recursos en abierto disponibles y accesibles en internet (wiki).

- Red social. Red social profesional en LinkedIn con más de 891 miembros (Linkedin, 2014).

\section{PLANTEAMIENTO DE LA INVESTIGACIÓN}

Se basa en dos fases: la primera fase consiste en crear el MOOC de software libre y conocimiento en abierto con un planteamiento que integre características de los modelos $\mathrm{X}$ y $\mathrm{C}$, de tal forma que genere recursos construidos de forma cooperativa por personas vinculadas al sector del software libre. La segunda fase consiste en utilizar esos recursos en la asignatura académica "Software libre" de tal forma que se pueda conocer el impacto en la misma.

\subsection{Primera fase}

La integración de los modelos X y C se basa en incorporar colaboraciones del mundo

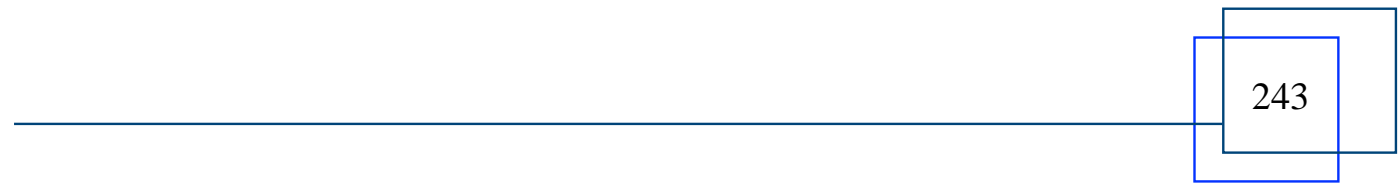




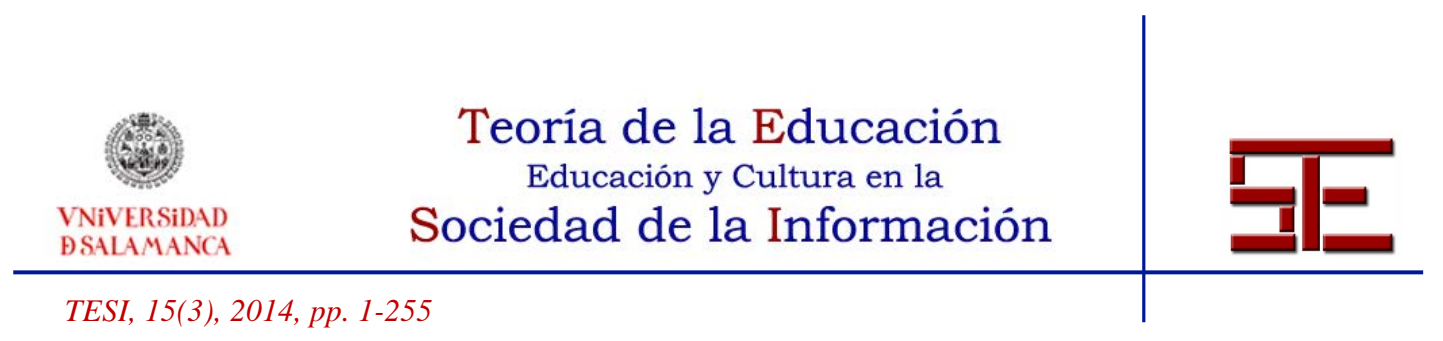

profesional, académico, laboral y social de la temática de la asignatura. Estas colaboraciones se han realizado a través de vídeos que grabaron los propios colaboradores o en el Gabinete de Tele Educación (GATE) de la Universidad Politécnica de Madrid. El profesorado organiza dichos recursos junto a los que elabora el propio equipo docente y toda la organización se realiza en la plataforma MiríadaX (MiríadaX, 2014), siguiendo un formato de curso tradicional.

El profesor sigue siendo organizador, asesor y guía, pero no posee el rol de tutor ni evaluador en su forma más clásica. Esto último se debe al alto número de alumnos matriculados y la imposibilidad de atender de forma personalizada a cada uno de ellos.

Para la modalidad de tipo C se incluyen, además, las redes sociales (LinkedIn, Twitter, indeti.ca y la red "Sociedad y Tecnología" (Elgg, 2014)) como medio para crear comunidades de aprendizaje. Desde el punto de vista formativo, el objetivo es que se produzca cooperación en la realización de actividades y, de esta forma, conseguir que se produzca aprendizaje. Se favorece el aprendizaje cooperativo, compartiendo recursos, ampliando el conocimiento a través de debates y propiciando la continuidad del proceso de aprendizaje tras finalizar el curso. Sin embargo, desde el punto de vista de la investigación, el objetivo es que se produzcan recursos que se puedan utilizar en la formación académica, formando parte, a su vez, de la comunidad de aprendizaje (red social y los recursos en abierto generados en la red social).

\subsection{Segunda fase}

Consiste en integrar, en la asignatura académica Software Libre, los recursos generados en el MOOC. El objetivo es que la comunidad de aprendizaje sirva como medio de colaboración entre el alumnado y el sector profesional y que, además, participe en la comunidad aportando recursos en abierto.

Para comprobar el resultado de satisfacción se empleará una adaptación del cuestionario SEEQ (Student's Evaluation of Educational Quality) creada por Hernert Marsh (1982) y que permite analizar la eficacia de la enseñanza utilizando una serie de factores, cada uno de los cuales consta de varios ítems, que son valorados en una escala tipo Likert de cinco opciones (muy en desacuerdo, en desacuerdo, ni de acuerdo ni en desacuerdo, de acuerdo y muy de acuerdo). La elección del cuestionario SEEQ está fundamentada en tres ventajas: sus propiedades psicométricas (Marsh, 1984), su amplia utilización en universidades de todo el mundo y la gran cantidad de material para el perfeccionamiento de cada uno de los ítems analizados (Verdugo y Cal, 2010, 3).

En este trabajo se incluyeron factores de valoración como: aprendizaje, entusiasmo, contenidos, organización, recursos incluidos y generados en el modelo con vistas al aprendizaje de conceptos $y$ objetivos de recursos incluidos y generados en el modelo con vistas a su aplicación.

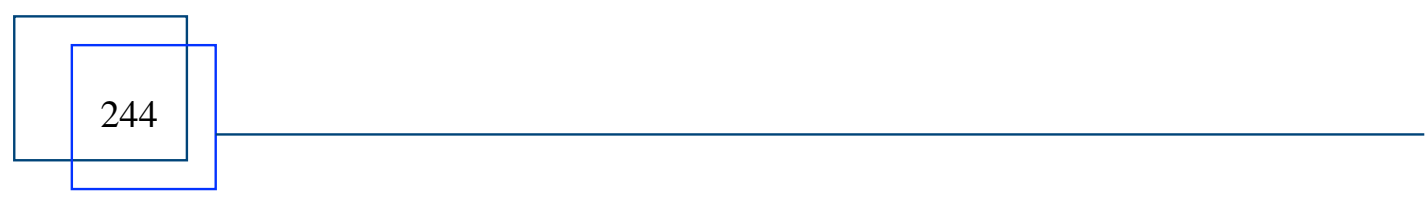




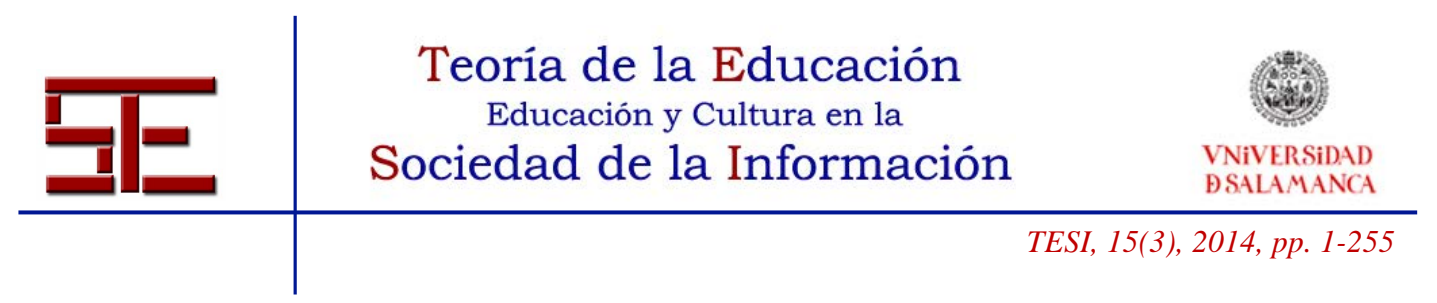

\section{RESULTADOS DE LA INCLUSIÓN DE LOS OER GENERADOS EN EL MOOC EN LA ASIGNATURA ACADÉMICA}

A continuación se muestran los resultados obtenidos durante las distintas fases del trabajo.

\subsection{Resultados fase 1. Productos generados en el MOOC}

En el MOOC se inscribieron 3.754 personas y se realizó una encuesta entre ellos para conocer, entre otros datos, su perfil profesional. Fue respondida por 1798 personas, un $47,89 \%$ de la muestra y los datos que arrojó la encuesta respecto al perfil profesional fueron: Docentes 13\%, Estudiantes 20\%, Sin actividad 21\% y diversas profesiones $41 \%$. Por tanto el perfil profesional es muy variado. Hay más representantes del sector no académico $41 \%$ que del sector académico (33\%).

Se generaron 4 redes sociales, dos propietarias y conocidas (LinkedIn y Twitter) y dos basadas en software libre (Elgg e identi.ca). Linkedin y Elgg son redes sociales clásicas, mientras que Twitter e identi.ca son redes sociales basadas en microbloggin (mensajes muy cortos). En la Tabla 2 se muestra el número de usuarios en cada red.

Tabla 2. Usuarios en las redes sociales.

\begin{tabular}{|l|l|}
\hline Red Social & Usuarios \\
\hline Linkedin & 891 \\
\hline Twitter & 200 \\
\hline Elgg & 76 \\
\hline Identi.ca & 90 \\
\hline
\end{tabular}

La red que más actividad tuvo, en cuanto a realizar aportes de recursos en abierto, fue LinkedIn. A partir de esos recursos y los que ya existían el curso se realizó un wiki (Wiki SL, 2014) donde se organizaron todos los recursos.

Así pues, el resultado de la realización del MOOC consistió en una red de 891 usuarios del curso y un wiki donde se organizaron todos los recursos, tanto previos como generados durante el curso por los participantes. 


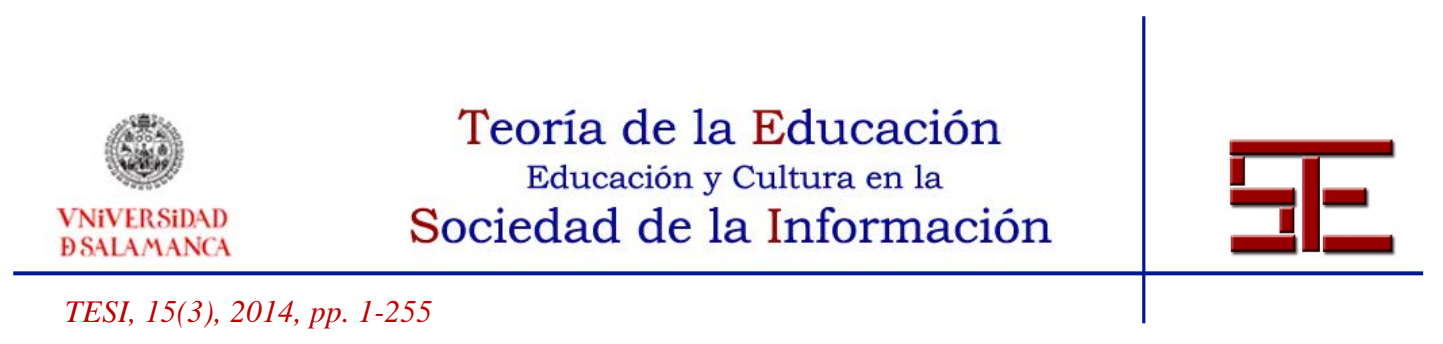

\subsection{Resultados fase 2. Utilización de los productos generados en la asignatura} académica

La asignatura Software libre es una asignatura de libre elección online y participa en un proyecto de colaboración entre la Universidad Politécnica de Madrid y la Universidad Autónoma de Barcelona, por lo que los estudiantes matriculados corresponden a ambas universidades.

Los recursos en abierto que se dispusieron en el curso se categorizaron como:

- Vídeos del profesor. Vídeos del MOOC. Recursos de aprendizaje donde el profesorado aporta conocimientos conceptuales de la temática de la Asignatura.

- Vídeos colaboradores. Vídeos del MOOC. Recurso aportados por personas con distintos perfiles y con distintas aplicaciones del software libre (organizaciones públicas, empresas de desarrollo informático, industria y usuarios).

- Wiki. Con los recursos en abierto aportados por los participantes del MOOC.

- Red social en LinkedIn. Red profesional donde usuarios comparten recursos, información y noticias sobre software libre y conocimiento en abierto.

Los objetivos formativos de la asignatura son idénticos a los años anteriores, es decir, no se variaron con la introducción de los nuevos recursos del MOOC.

La muestra, utilizada en el cuestionario SEEQ, fueron los estudiantes matriculados en la asignatura Software libre, 41 de la Universidad Politécnica de Madrid y 20 de la Universidad Autónoma de Barcelona. El número de estudiantes que contestó a la encuesta ha sido de 49 , lo que hace un $80,32 \%$ del total.

En relación a las preguntas de la encuesta, se observa que el 79\% de los estudiantes eran del género masculino, acorde con los Grados en Ingeniería. Como dato a resaltar, la distribución del alumnado por género en el MOOC tuvo unos resultados similares, un $72 \%$ de género masculino y un $28 \%$ de género femenino.

Por otra parte, el alumnado pertenece a muy distintas titulaciones y centros formativos. Por ese motivo se ha agrupado el perfil de procedencia por universidad. Siendo mayoritarios los estudiantes de la Universidad Politécnica de Madrid (71\%) entre los que cumplimentaron la encuesta, los restantes son de la Universidad de Barcelona.

Respecto a los objetivos de los recursos incluidos y generados en el modelo, señalamos dos tipos: los de aprendizaje de conceptos (objetivo: dar una visión general del Software libre y Conocimiento en abierto) y los objetivos aplicados (objetivo: participar en el movimiento de Software libre y Conocimiento en abierto). Las valoraciones de los recursos contenidos (vídeos internos y externos, red social y wiki) sigue la escala Likert

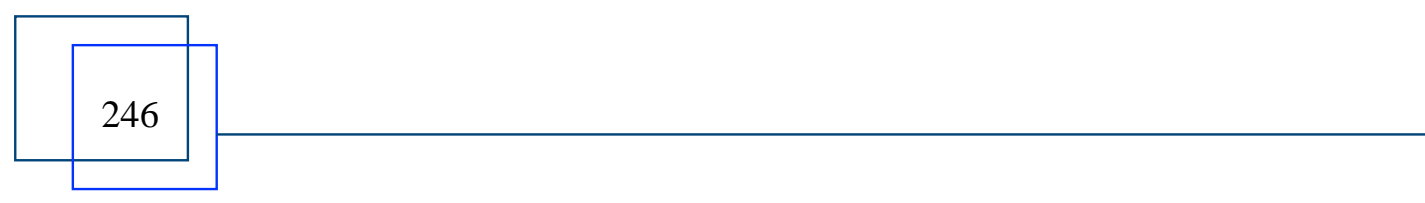




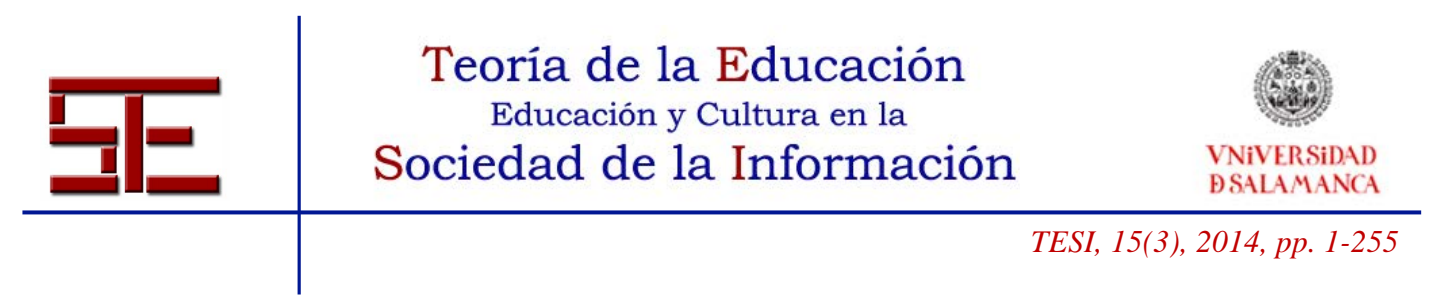

(1- Nada hasta 5- Mucho) y se encuentran todas entre 4 y 5 , para cualquiera de los dos objetivos (Fig. 3 y 4). En general, todos los recursos están bien valorados. Sin embargo, los recursos más valorados para el aprendizaje conceptual es el del profesorado, mientras que para la aplicación de dichos conceptos hay una distribución más homogénea y son igual de valorados los recursos aportados por los miembros del MOOC (el wiki) que los del profesorado. La red social también es prácticamente igual de valorada.

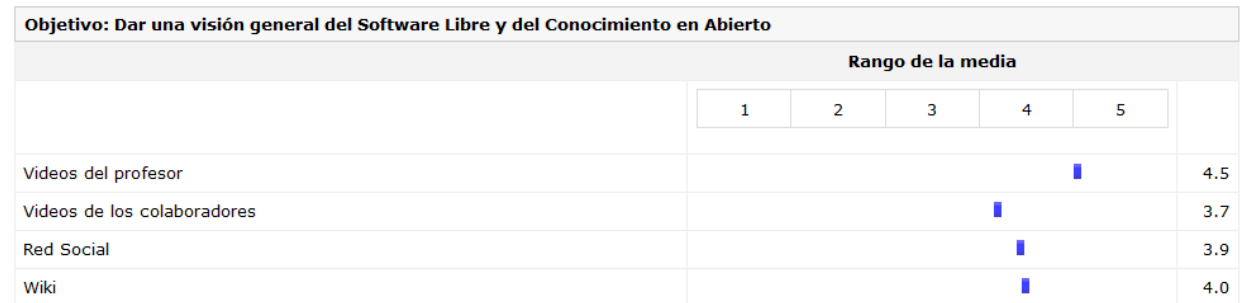

Figura 3. Valoración de los recursos incluidos para aprendizaje de conceptos.

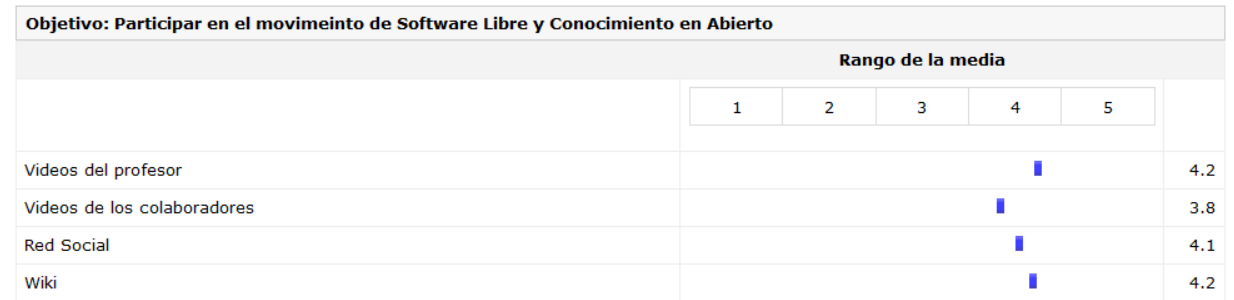

Figura 4. Valoración de los recursos incluidos para aplicación de conocimiento.

En la Figura 5 se observa la elevada valoración del alumnado respecto a su aprendizaje. La valoración más alta $(4,6)$ corresponde a su percepción de la comprensión de los contenidos del curso (He aprendido y comprendido los contenidos del curso). El resto de ítems son: en este curso he aprendido cosas que considero valiosas, mi interés por los temas tratados en este curso ha aumentado al realizar el curso y el curso me ha parecido estimulante.

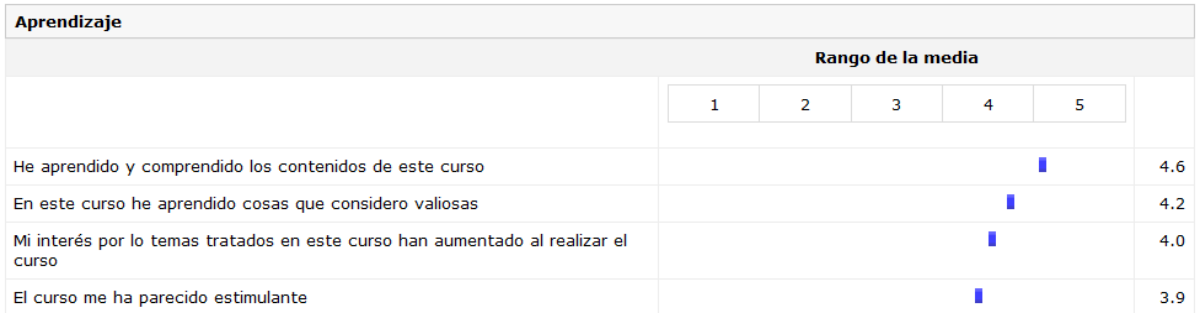

Figura 5. Valoración del aprendizaje. 


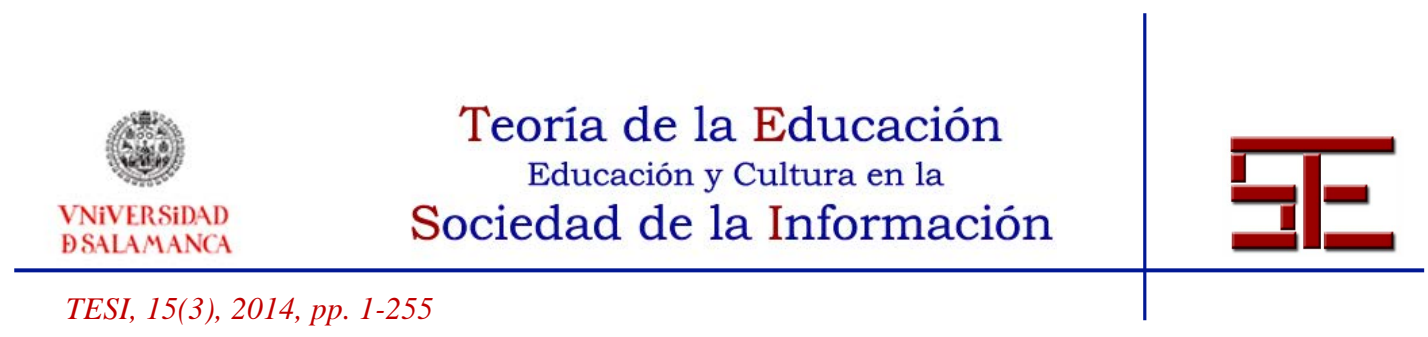

Respecto al entusiasmo, en general es muy homogéneo en cuanto a la motivación y dinámica, y todas las respuestas se encuentran en el valor 4 (ver Figura 6). Las preguntas fueron: el curso ha sido dinámico y activo, la forma de presentar los módulos consiguió mantener mi atención, en general los vídeos incluidos en el curso son amenos, el profesorado que ha grabado los vídeos ha mostrado entusiasmo (la más valorada), he participado con regularidad y trabajo activamente en el curso. Estas valoraciones están acorde con las obtenidas en la valoración del aprendizaje, donde el interés y el estímulo por la asignatura están presentes.

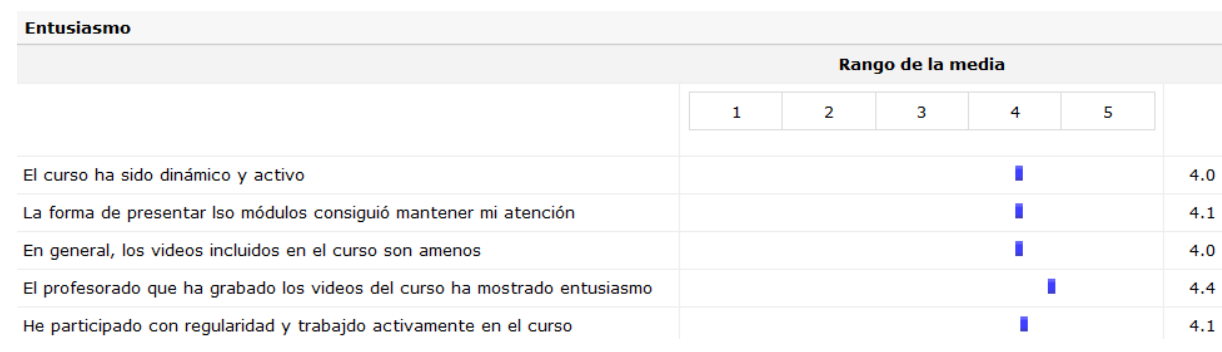

Figura 6.Valoración del entusiasmo.

En la Figura 7 se muestra que, en general, los recursos aportados por el profesorado, la realización de los vídeos y la claridad y apoyo de los mismos, son más valorados que los videos aportados por los colaboradores. Sin embargo los recursos generados por los participantes en el MOOC están muy bien valorados.

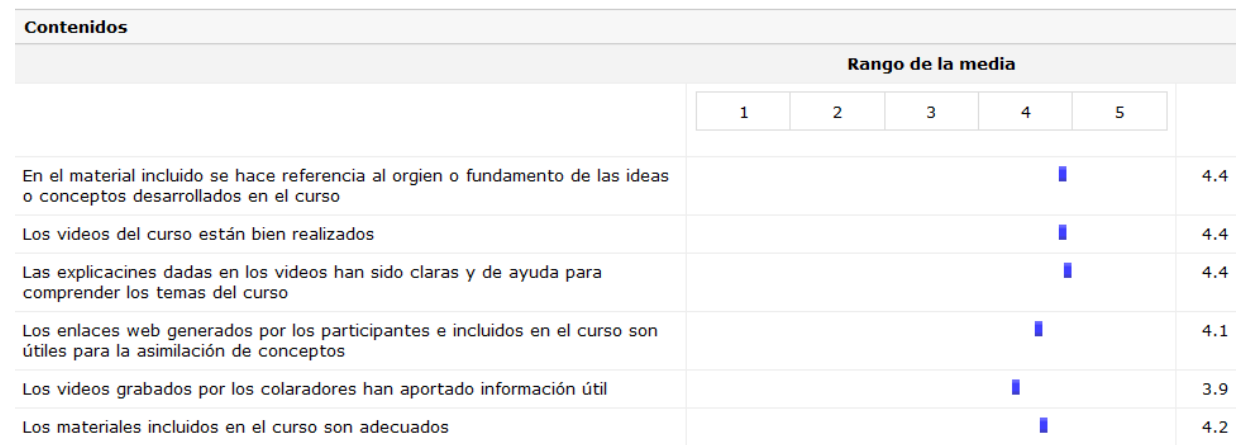

Figura 7. Valoración de los contenidos. 


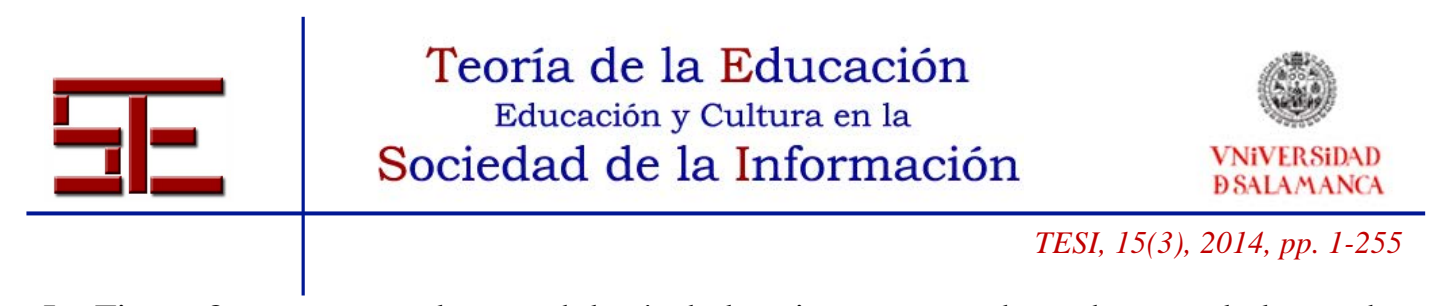

La Figura 8 muestra que la metodología de la asignatura es adecuada para el alumnado, ya que siempre se supera el valor 4 para todos los ítems. Se encuentra valoración especial en la posibilidad de utilizar los recursos una vez finalizado el curso.

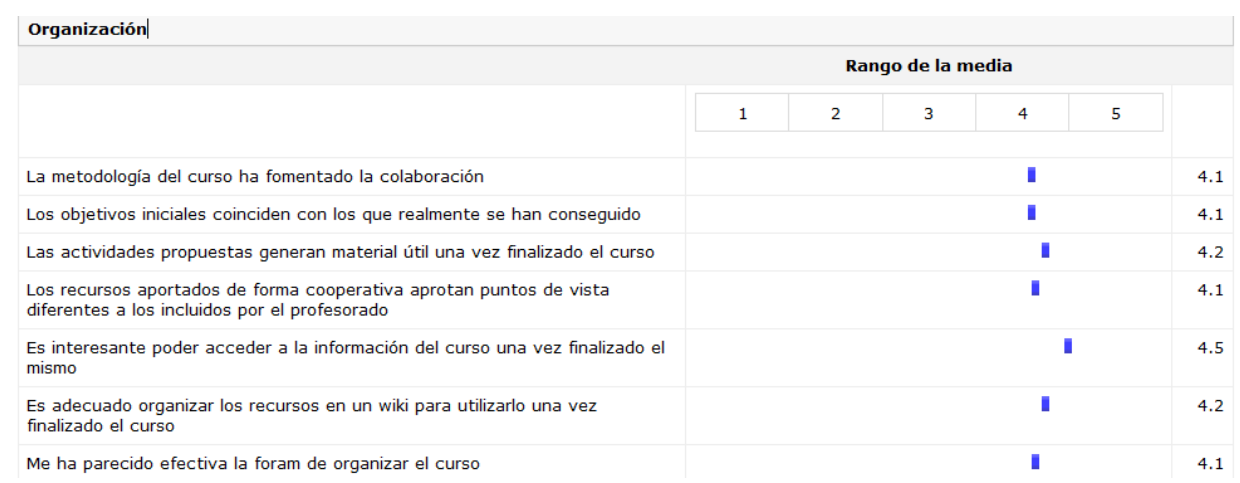

Figura 8. Valoración de la organización de los contenidos.

\section{CONCLUSIONES Y TRABAJO FUTURO}

Los resultados, tanto cuantitativos como cualitativos, indican que es posible integrar la formación académica con el modelo MOOC de formación en abierto.

La integración principal se realiza a través de los recursos aportados por el profesorado y los aportados por los estudiantes del MOOC.

La percepción del alumnado de la asignatura académica es favorable, estando siempre en puntuaciones cercanas al valor 4 sobre 5 .

El modelo en espiral establece una interacción entre los cursos aunque no coincidan los periodos de inicio y final. La comunidad de aprendizaje es un elemento que tiene "vida propia", por tanto se puede utilizar en cualquier lugar y momento. Esto permite que la asignatura académica interactúe tanto con las personas como con los recursos generados por los participantes en el MOOC.

Se ha generado un modelo con interacciones entre OER de distintas iniciativas y la asignatura académica; estableciendo unos flujos continuos de conocimiento en abierto y una comunidad de aprendizaje donde se incluyen alumnos de la universidad, alumnos del MOOC, usuarios de OCW y personas relacionadas con la temática de la asignatura.

El modelo propuesto presenta las siguientes características:

- Creación de una comunidad de aprendizaje (compuesta por redes sociales y

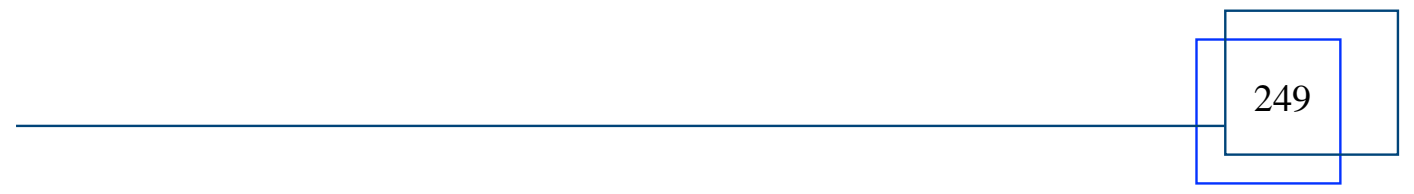




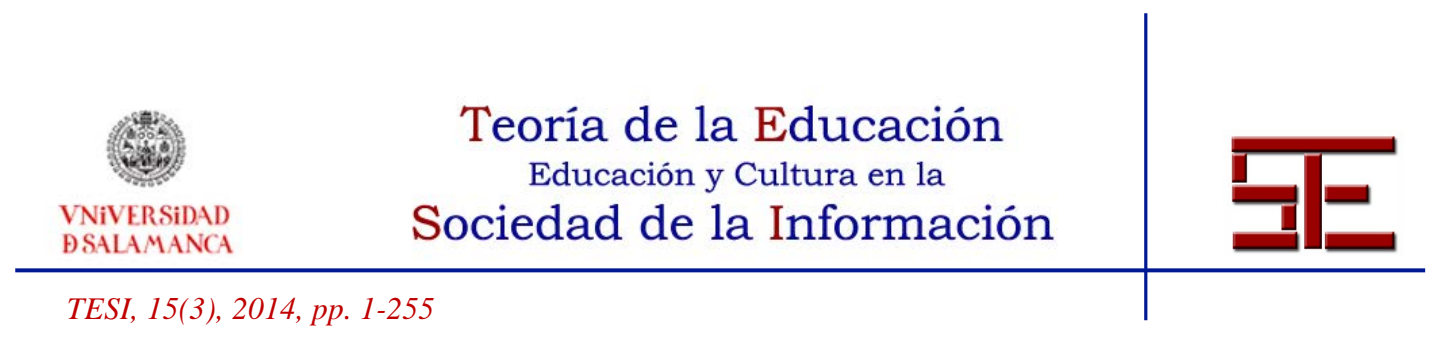

wikis) compartida por OER de distintas iniciativas OER (OCW, MOOC y Web Social), la asignatura académica y el contexto profesional.

- Actualización continua de conocimiento generado por la comunidad de aprendizaje.

- Reutilización de Los OER por las distintas iniciativas.

- Sostenibilidad de los OER y mejora continua de los mismos.

- Dotación, a la asignatura OCW, de flujos dinámicos y comunidad de aprendizaje.

Con todo lo anterior, se dota a la asignatura académica de elementos propios del movimiento OER.

- Cultura cooperativa y participativa.

- Integración con el mundo profesional, laboral y social.

- Participación en un servicio público a través de los OER

- Participación del profesorado y alumnado en el movimiento OER de forma activa.

Se ha demostrado que, a través del MOOC, se pueden crear comunidades de aprendizaje con personas pertenecientes al mundo académico, profesional y social. Se trata, por tanto, de aprovechar esta circunstancia e integrarla tanto con el resto de iniciativas OER como con la asignatura académica.

El alumnado de la asignatura académica participó de forma activa en la comunidad de aprendizaje, uniéndose a la red social y aportando recursos al wiki. Como línea futura se estudiará la influencia de esta participación en el siguiente MOOC que realizará el equipo de trabajo. Además se está trabajando en metodologías y tecnologías que faciliten la gestión adaptativa (Fidalgo et al., 2013a) del conocimiento generado en las iniciativas OER que componen este modelo (Sein-Echaluce et al., 2013). El objetivo es demostrar que el acceso a ese conocimiento generado contribuye a la mejora del aprendizaje, durante la realización del curso y una vez finalizado el mismo.

\section{AGRADECIMIENTOS}

Los autores quieren hacer constar el agradecimiento al Gabinete de Tele-Educación de la Universidad Politécnica de Madrid por su apoyo en la puesta en marcha del curso

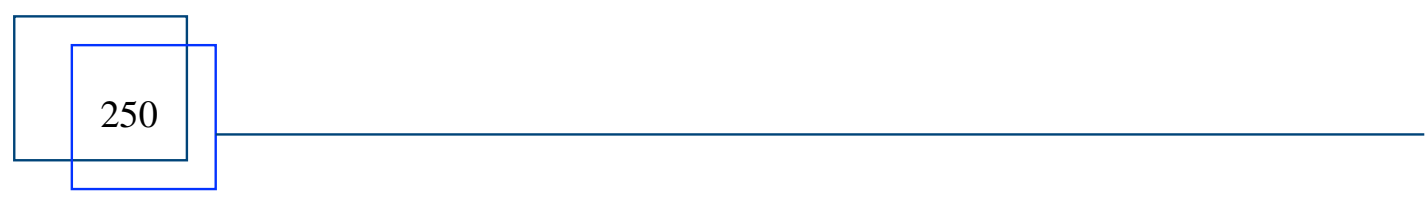




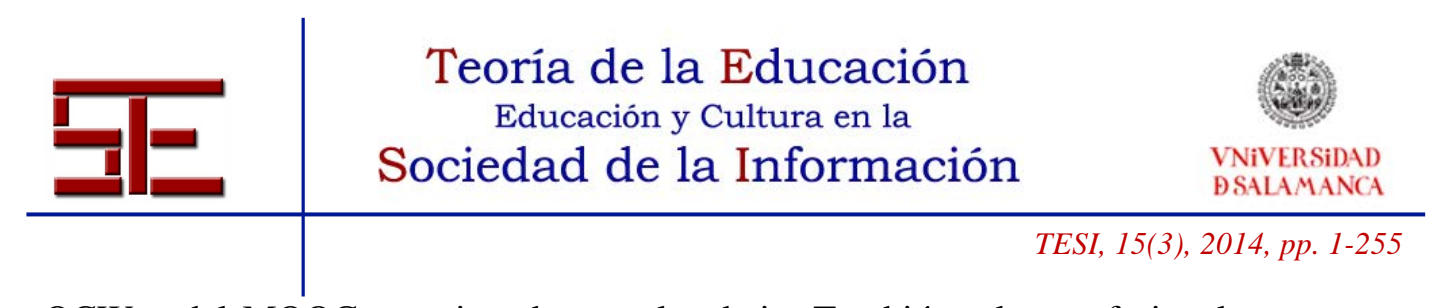

OCW y del MOOC mencionados en el trabajo. También a los profesionales externos que aportaron sus experiencias para enriquecer los contenidos del MOOC. Así como al Gobierno de Aragón, al Fondo Social Europeo y a la Junta de Castilla y León por su apoyo. Finalmente los autores quieren agradecer el apoyo de sus grupos de investigación (LITI, <http://www.liti.es>; GIDTIC, <http://gidtic.com> y GRIAL, $<$ http://grial.usal.es>).

\section{REFERENCIAS}

ADA-Madrid (2014). Consultado el 10 de junio de 2014 <http://moodle.upm.es/adamadrid/>.

Aps (1993). U.S. History.com. Civilian Conservation Corps (CCC) 1933-1941.

Borrás, O. (2012). Píldoras formativas y videojuegos aplicados al estudio de la Ingeniería Acústica. Tesis (Master). Madrid: E.T.S.I. Telecomunicación (UPM).

BSCW (2014). Consultado el 10 de junio de 2014. https://public.bscw.de/pub/

CNI (2008). El conocimiento libre y los Recursos Educativos Abiertos. Coord. Centro de Nuevas Iniciativas. Ed. OECD y Junta de Extremadura. Último acceso 10 junio 2014 <http://www.oecd.org/edu/ceri/42281358.pdf >.

Downes, S. (2008) MOOC and Mookies: The Connectivism \& Connective Knowledge Online Course. Consultado el 10 de junio de 2014. $<$ http://www.slideshare.net/Downes/mooc-and-mookiesthe-connectivism-connectiveknowledge-online-course-presentation>.

Educational Resources. Consultado el 10 de junio de 2014 <http://jime.open.ac.uk/article/2012-10/html>

Elgg (2014). Consultado el 10 de junio de 2014. <http://www.sociedadytecnologia.org/groups/profile/187610/mooc-softwarelibre-yconocimiento-en-abierto>.

Fidalgo, Á., García-Peñalvo, F. J., y Sein-Echaluce, M. L. (2013a). A methodology proposal for developing adaptive cMOOC. In F. J. García-Peñalvo (Ed.), Proceedings of the First International Conference on Technological Ecosystem for Enhancing Multiculturality (TEEM'13), 553-558. New York, NY, USA: ACM.

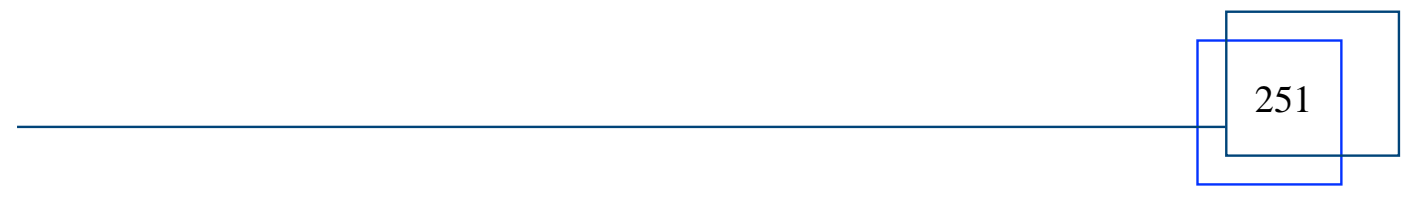




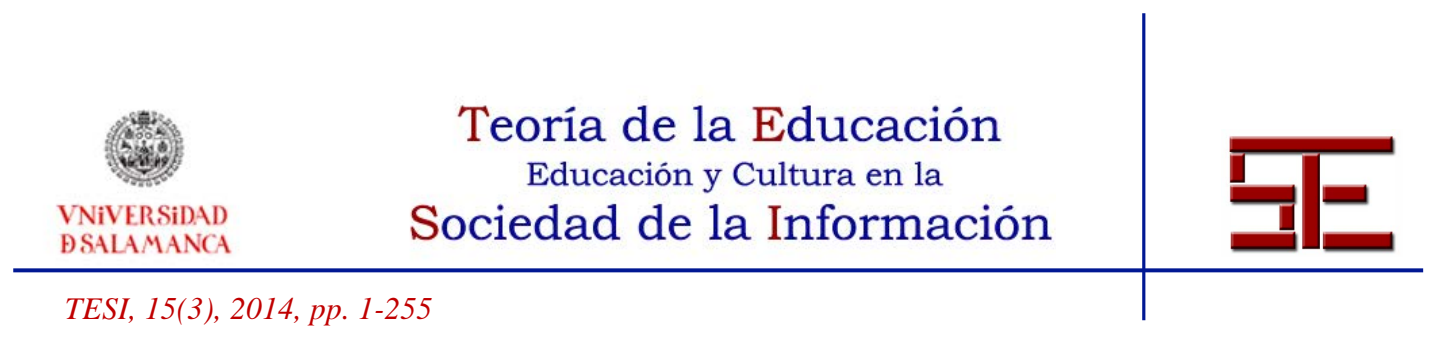

Fidalgo, A., Sein-Echaluce, M. L., y García Peñalvo, F. J. (2013b). MOOC cooperativo. Una integración entre cMOOC y xMOOC. En: Libro de Actas CINAIC 2013. pp. 481 486. Consultado el 10 de junio de 2014. <http://www.dmami.upm.es/dmami/documentos/liti/ACTAS_CINAIC_2013.pdf>.

García-Peñalvo, F. J. (2005). Estado actual de los sistemas E-Learning. Teoría de la Educación. Educación y Cultura en la Sociedad de la Información, 6(2).

García-Peñalvo, F. J. (2008). Advances in E-Learning: Experiences and Methodologies. Hershey, PA, USA: Information Science Reference (formerly Idea Group Reference).

García-Peñalvo, F. J., García de Figuerola, C., y Merlo, J. A. (2010a). Open knowledge management in higher education. Online Information Review, 34(4), 517-519.

García-Peñalvo, F. J., García de Figuerola, C., y Merlo, J. A. (2010b). Open knowledge: Challenges and facts. Online Information Review, 34(4), 520-539. doi: $10.1108 / 14684521011072963$

Holmes, B. (2006). Quality in Europe of diverse systems and sharing golas. En: Ehlers, Ud \& Pawlowski, J. (eds). Handbook on quality and standardization en e-learning. Berlin: Springer.

Khan (2006). Consultado el 10 de junio de 2014. 〈http://www.khanacademy.org>.

Lane, A. (2010) eDesigning for innovation around OER. Journal of interactive Media in Education. Special Issue on Open Educational Resources. Consultado el 10 de junio de 2014 http://jime.open.ac.uk/article/2010-2/html

Liddo, A., Buckingham Shum, S., McAndrew, P., y Farrow, R. (2012) The open education evidence hub: A collective intelligence tool for evidence based policy. En: Cambridge 2012: Joint OERI2 and OpenCourseWare Consortium Global 2012 Conference, 16 - 18 April 2012, Cambridge, UK.

Linkedin (2014) Software libre y conocimiento en abierto (Grupos). Consultado el 10 de Junio de 2014. <https://www.linkedin.com/groups?home=\&gid=4889669\&trk=anet_ug_hm>

Margulies, A. (2005) MIT Opencourseware - A New Model for Open Sharing. Conferencia OpenEd en la Utah State University, Septiembre.

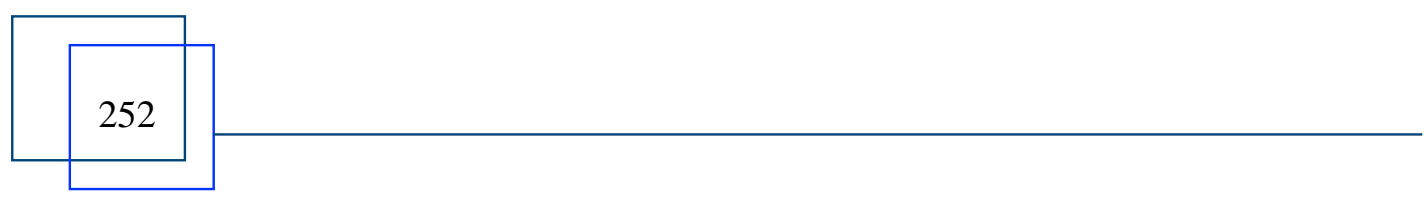




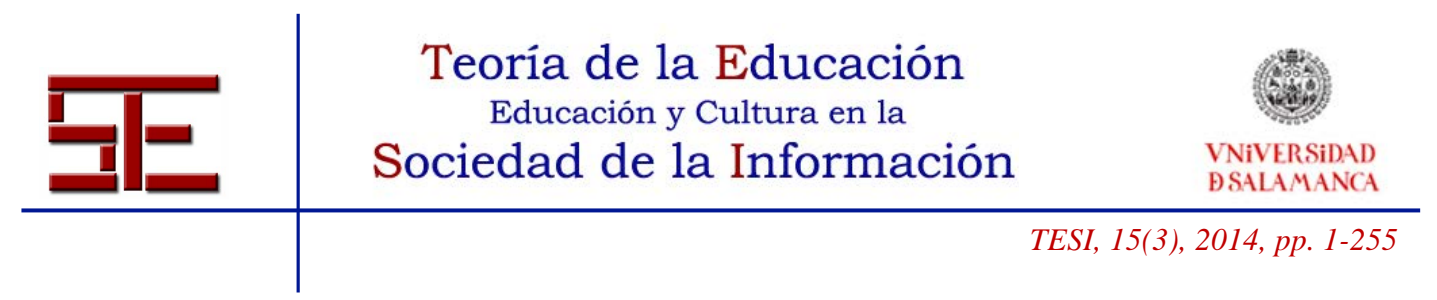

Markoff (2011). Virtual and Artificial, but 58,000 Want Course. Consultado el 10 de junio de 2014. <http://www.nytimes.com/2011/08/16/science/16stanford.html?_r=1\&>.

Marsh, H. (1982). SEEQ: A reliable valid and useful instrument for collecting students evaluations of university teaching. British Journal of Educational Psychology, 52, 7-95

Marsh, H. (1984). Students Evaluations of University Teaching: Dimensionality, Reliability, Validity, Potential Bias and utility. Journal of Educational Psychology, 76, 707-754.

McAndrew P. (2012). Learning the lesssons of Openness. Journal of interactive Media in Education. Special Issue on Open.

McAndrew, P. (2010). Researching open content - experiences from the OpenLearn initiative. Consultado el 10 de junio de 2014. <http://kn.open.ac.uk/public/document.cfm?docid=13201>.

MiríadaX (2014). Último acceso 10 junio 2014. <http://miriadax.net>.

MIT (2014). Último acceso 10 junio 2014. <http://ocw.mit.edu/index.htm>.

MOOC (2012). Último acceso $10 \quad$ junio 2014. <http://miriadax.net/web/soft_libre_y_conocimiento>.

Moodle (2014). Último acceso 10 junio 2014. 〈http://www.moodle.org>.

Moore, A. (2002) Lens on the Future: Open-source Learning. Educause Review, 37(5)

NetMeeting (2014). Último acceso $10 \quad$ junio 2014. <http://support.microsoft.com/kb/154143/es>.

OCW (2008). I Premio MEC-Universia a la mejor asignatura del año en OCW. Último acceso 10 de junio de 2014. <http://noticias.universia.es/vidauniversitaria/noticia/2008/05/26/580377/conoce-equiposganadores-premio-mecuniversia-reconoce-mejor-asignatura-ano-ocw.html>.

OCWC (2014). OpenCourseWare Consortium. Último acceso 10 junio 2014. <http://www.oeconsortium.org>.

OECD (2007). Giving Knowledge for free: the emergence of open educational resources. Último acceso 10 de junio de 2014.

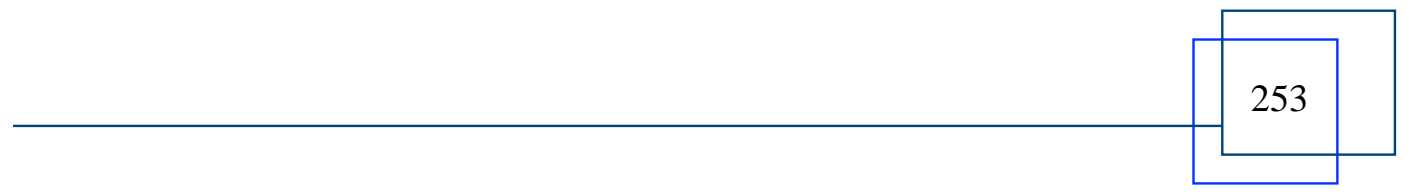




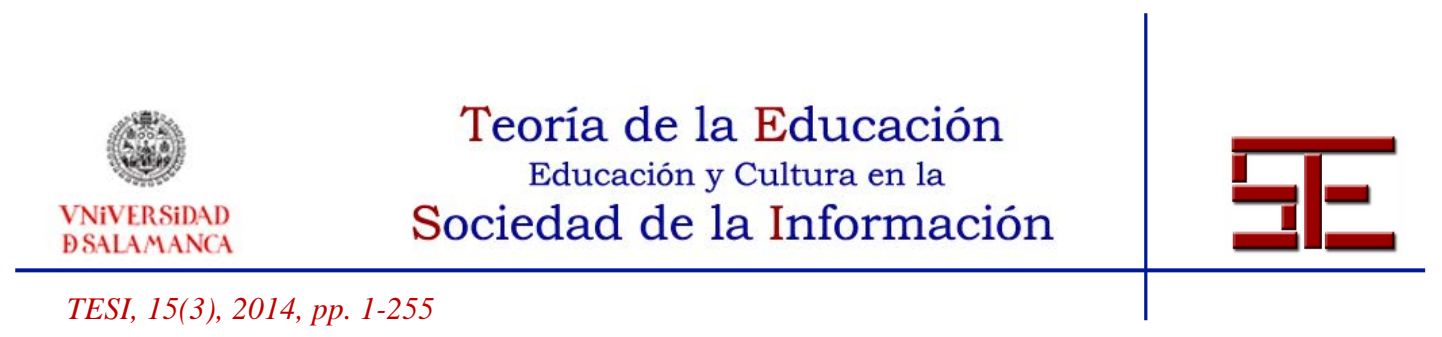

$<$ http://www.oecd.org/edu/ceri/givingknowledgeforfreetheemergenceofopeneducational resources.htm>.

Ossiannilsson, E., y Creelman, A. (2011). Quality improvement of the use of OER in higher education - challenges and consequences. European Association of Distance Teaching Universities Annual. Conference. Último acceso 10 de junio 2014. <http://lnu.diva-portal.org/smash/get/diva2:469226/FULLTEXT01>.

Piedra (2010). An Approach for Description of Open Educational Resources based on Semantic Technologies. En: Education Engineering (EDUCON), 2010 IEEE. 11111119.

Pisutova. K. (2012). Open Education. 10th IEEE International Conference on Emerging eLearning Technologies and Applications. November 8-9.

Sein-Echaluce, M. L., Lerís, D., Fidalgo Blanco, Á., y García-Peñalvo, F. J. (2013). Knowledge management system for applying educational innovative experiences. En F.

J. García-Peñalvo (Ed.), Proceedings of the First International Conference on Technological Ecosystem for Enhancing Multiculturality (TEEM'13) (pp. 405-410). New York, USA: ACM.

Siemens (2006). Knowing Knowledge. Último acceso 10 de junio de 2014. <http://www.elearnspace.org/KnowingKnowledge_LowRes.pdf >.

SL (2007). Asignatura "Software libre" en OCW de la UPM. Último acceso 10 de junio de 2014. <http://ocw.upm.es/ciencia-de-la-computacion-e-inteligenciaartificial/software-libre>.

UNESCO (2010) Taking OER beyond the OER Community: Policy and Capacity. UNESCO/Commonwealth of Learning policy forum. Paris, France. Último acceso 10 junio 2014. http://oerworkshop.weebly.com/uploads/4/1/3/4/4134458/taking_oer_beyond_the_oer_ community_policy_forum_final.pdf

UNESCO (2011a). Guidelines for open educational resources OER in higher education. $\begin{array}{llll}\text { Último } & \text { acceso } & 10 & \text { junio }\end{array}$ $<$ http://www.col.org/resources/publications/Pages/detail.aspx?PID=364>.

UNESCO (2011b) A Basic Guide to Open Educational Resources (OER). Unesco Commonwealth of Learning. Último acceso 10 junio 2014.

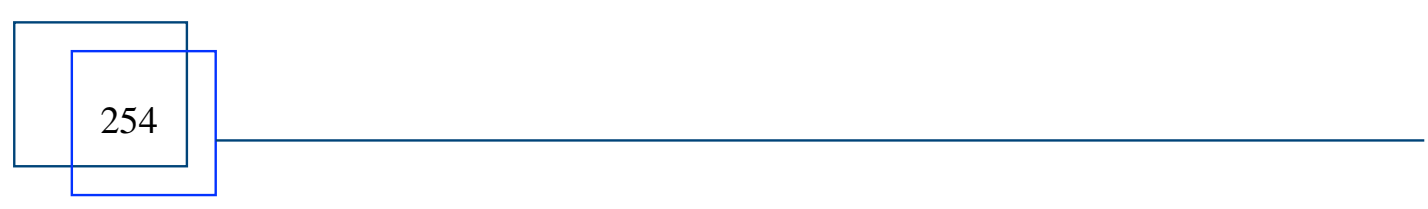




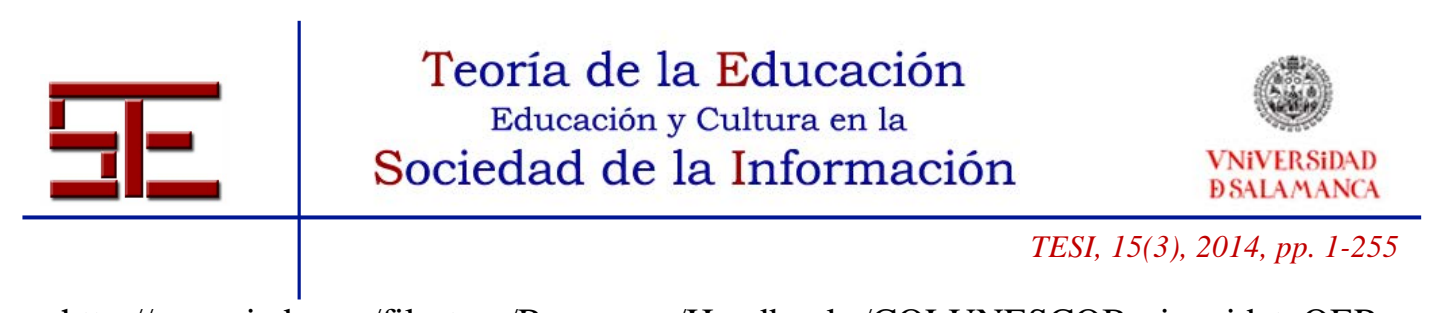

<http://www.icde.org/filestore/Resources/Handbooks/COLUNESCOBasicguidetoOER. pdf>.

UNESCO (2012) 2012 Paris OER Declaration. Último acceso 10 junio 2014. <http://www.unesco.org/pv_obj_cache/pv_obj_id_EEF3C7E6694B8B91C31F5EA3340 D484EF03A0100/filename/Paris\%20OER\%20Declaration_01.pdf>.

Verdugo, V., y Cal, M. A. (2010). Valoración de la enseñanza, SEEQ Revista de Formación e Innovación Educativa Universitaria. 3(4), 182- 193. Último acceso 10 de junio de 2014. 〈http://webs.uvigo.es/refiedu/Refiedu/Vol3_4/REFIEDU_3_4_2.pdf>.

Wiki (2014). Último acceso 10 de junio de 2014. <http://creandowikis.wikispaces.com>.

Wiki SL (2014). Wiki creado de forma colaborativa sobre "Software libre". Último $\begin{array}{llll}\text { acceso } & 10 & \text { junio } & 2014 .\end{array}$ libre.shoutwiki.com/wiki/P\%C3\%A1gina_principal>.

Wiley, D. (2006) The Learning Objects Literature. Chapter 29. 345-352. Último acceso 10 junio 2014 <http://www.opencontent.org/docs/wiley-lo-review-final.pdf〉.

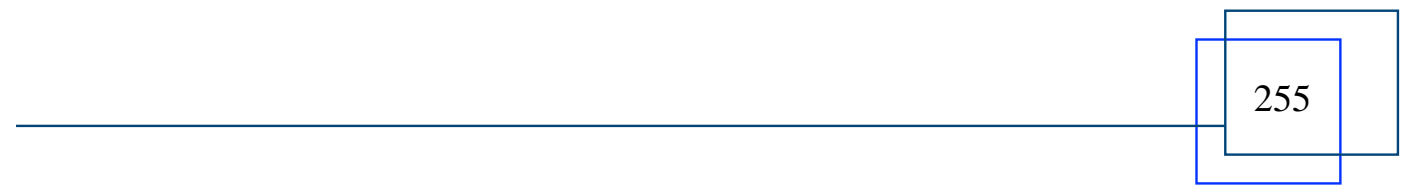

